\title{
Upward high-energy field-aligned electron beams above the polar edge of auroral oval: observations from the SKA-3 instruments onboard the Auroral Probe (Interball-2)
}

\author{
V. A. Stepanov ${ }^{1}$, Y. I. Galperin ${ }^{1}$, A. K. Kuzmin ${ }^{1}$, F. K. Shuiskaya ${ }^{1}$, L. S. Gorn ${ }^{2}$, B. A. Ilyin ${ }^{2}$, M. V. Iovlev², \\ A. A. Klimashov ${ }^{2}$, I. I. Cherkashin ${ }^{2}$, B. I. Khazanov ${ }^{2}$, and A. Y. Safronov ${ }^{2}$ \\ ${ }^{1}$ Space Research Institute of Russian Academy of Sciences, Profsoyuznaya str., 84/32, Moscow 117810, Russia \\ ${ }^{2}$ Scientific Engineering Center (SNIIP), Raspletina str., 5, Moscow 123060, Russia
}

Received: 25 August 1997 / Revised: 30 April 1998 / Accepted: 12 May 1998

\begin{abstract}
A new phenomenon was found at the polar edge of the auroral oval in the postmidnight-morning sectors: field-aligned (FA) high-energy upward electron beams in the energy range $20-40 \mathrm{keV}$ at altitudes about $3 \mathrm{R}_{\mathrm{E}}$, accompanied by bidirectional electron FA beams of $\mathrm{keV}$ energy. The beam intensity often reaches more than $0.5 \cdot 10^{3}$ electrons $/ \mathrm{s} \cdot \mathrm{sr} \cdot \mathrm{keV} \cdot \mathrm{cm}^{2}$, and the beams are observed for a relatively long time $\left(\sim 3 \cdot 10^{2}-10^{3} \mathrm{~s}\right)$, when the satellite at the apogee moves slowly in the ILAT-MLT frame. A qualitative scenario of the acceleration mechanism is proposed, according to which the satellite is within a region of bidirectional acceleration where a stochastic FA acceleration is accomplished by waves with fluctuating FA electric field components in both directions.
\end{abstract}

Key words. Ionosphere (particle acceleration; waveparticle interactions) - Magnetospheric physics (magnetosphere-ionosphere interactions)

\section{Introduction}

The Interball Auroral Probe carries the SKA-3 experiment whose goal was to investigate high energy particle intensities at high latitudes (i.e. above the auroral oval and inside the northern polar cap). In several cases at the polar edge of the oval in the postmidnight - morning sectors in the energy range $20-40 \mathrm{keV}$ the SKA-3 instruments detected field-aligned high-energy upward electron beams (see Galperin, 1997; Stepanov et al., 1997). These beams were observed as field-aligned (FA) monodirectional (upward), while simultaneous measurements made by the ION spectrometer (data courtesy of

Correspondence to: Y. I. Galperrin
J.-A. Sauvaud and R.A. Kovrazhkin, see Sauvaud et al., this issue) showed that the $\mathrm{keV}$ energy electron fluxes were bidirectional. In some cases, upward accelerated ions $\left(\mathrm{O}^{+}\right)$of $100-1000 \mathrm{eV}$ energy (at least partly folded ion conics) were detected simultaneously with the SKA3 upward electron beam detection. For one case, upward beam of low-energy (about $10 \mathrm{eV}$ ) $\mathrm{H}^{+}$was detected by the onboard thermal mass-spectrometer HYPERBOLOID (data courtesy of N. Dubouloz). However, interesting features of the low-energy ions observed simultaneously with the upward high-energy electron beams are beyond the scope of this study.

Several authors have reported observations of the upward electron beams in auroral regions (e.g., see Burch et al., 1983; and references therein). Hultqvist et al., 1988 were the first to observe relatively energetic (up to $8 \mathrm{keV}$ ) bidirectional field-aligned electron beams at altitudes $\sim 2 \mathrm{R}_{\mathrm{E}}$, i.e., above the main region of downward auroral electron acceleration. They have presented measurements from VIKING of upward moving electrons and ions of comparable energy at altitudes about $13500 \mathrm{~km}$ in the invariant latitude (ILAT) - magnetic local time (MLT) sector of $76^{\circ}$ and $\sim 9 \mathrm{~h}$, respectively, where the field lines were most probably closed, thus implying the possibility of electron bouncing. Observations were made during quite magnetic conditions after a period with several substorms. The upward electron beams detected by VIKING had a conical distribution at energies of several $\mathrm{keV}$, while at the lowest energies they were clearly beamed, and those electron conics were much narrower than ion conics. The lower energy electrons had much higher fluxes than those at high energies. It was suggested that the distributions of low-energy and high-energy electron beams were produced by different processes. Authors have proposed an interpretation in which the upward field-aligned acceleration of both electrons and ions was due to the fluctuating electric field with its mean parallel component different from zero.

The SKA-3 observations of energetic upward electron beams, presented in this work, in some aspects are 
similar to those reported by Hultqvist et al. 1988, but at the same time are quite distinct from them. We present a case study of upward electron beams in the energy range $20-40 \mathrm{keV}$ only, i.e., at energies which are substantially higher than typical auroral electron energies. Four orbits in the postmidnight sector were chosen for a detailed analysis during which the invariant latitude of the Auroral Probe exceeded $75^{\circ}$.

It is emphasized in this work that the upward acceleration of electrons to tens of $\mathrm{keV}$ observed by the Auroral Probe at altitudes of about $3 \mathrm{R}_{\mathrm{E}}$ at, or close to the polar boundary of the auroral oval, cannot be significantly influenced by a parallel quasi-DC electric field component and conjugate effects. These observations imply acceleration processes distributed in a wide altitude interval, both below and above the satellite apogee $\left(\sim 4 \mathrm{R}_{\mathrm{E}}\right.$ geocentric), caused by wave turbulence which leads to a stochastic particle acceleration. This means that during the upward electron beam registrations, the satellite at its apogee was within such extended regions of intense plasma turbulence and auroral FA acceleration.

\section{The SKA-3 instrumentation set}

The SKA-3 set of particle detectors installed onboard the Auroral Probe is aimed to measure low and medium energy electrons and ions. It consists of two energyangle analyzers EA-2 and EA-3 for low energy particles, and two identical oppositely directed time-of-flight (TOF) analyzers EM-1-1 and EM-1-2 for high energies. A short description of the instruments is given (for more information see Shuiskaya et al., 1995, 1998).

All instruments are mounted on the back of the satellite, opposite to Sunward direction, with fields of view perpendicular to the satellite rotation axis (which is Sunward directed). The satellite spinning thus scans the range of the pitch angles depending on the angle between the Sunward direction and magnetic field. The spin period of the satellite is $120 \mathrm{~s}$. Temporal resolution of observation modes varies from 0.1 to $0.8 \mathrm{~s}$, spatial resolution at apogee is about $0.5 \div 10 \mathrm{~km}$.

\subsection{Analyzers of low-energy charged particles (EA-2 and EA-3)}

EA-2 and EA-3 are energy-angle spectrometers based on electrostatic toroidal analyzers. Analyzers differ in polarity of voltages applied to systems of electrostatic deflection and microchannel plates (MCP) circuitry. EA-2 measures energy-angle distributions of electrons, EA-3 measure ion distributions, During a satellite rotation each window (of $22.5^{\circ}$ width with a gap of $22.5^{\circ}$ in between, and $5.7^{\circ}$ height) scans all the phase angle range. The instruments allow us to measure simultaneously in eight angular sectors evenly distributed at the angle $2 \pi$ perpendicular to the satellite rotation axis, and sequentially in seven energy intervals for each angular sector. The central energies of energy intervals are as follows: $30,85,240,680,1900,5300$, $15000 \mathrm{eV}$, the energy resolution $\Delta \mathrm{E} / \mathrm{E}$ is $10 \%$. Particles are recorded by means of the MCPs with spaced anodes for eight angular directions. In order to improve the angular resolution of the detector, a mask is applied to the MCPs corresponding to location of anodes, and a special sectioned collimator is attached to the entrance aperture. The calculated geometric factor is $6 \cdot 10^{-3} \mathrm{~cm}^{2} \cdot \mathrm{sr}$.

\subsection{Analyzers of medium-energy charged particles (EM-1-1 and EM-1-2)}

The instruments EM-1-1 and EM-1-2 are identical timeof-flight solid-state energy-mass spectrometers. Electrons are deflected from the ion aperture by a magnet. Ions are selected with masses, 1, 4 and 16 in seven energy intervals within energy range from 20 to $500 \mathrm{keV}$. Spectrometers are based on a combination of TOF device with a thick silicon semiconductor detector of the full energy at the end. Fields-of-view of these detectors are directed oppositely and are perpendicular to the satellite rotation axis. Each field-of-view is a cone of full angle $8^{\circ}$. Table 1 presents the ranges of energy intervals for each type of particles. For the $20-40 \mathrm{keV}$ electrons the geometric factor is $5.7 \cdot 10^{-2} \mathrm{~cm}^{2} \cdot \mathrm{sr}$, and $3 \cdot 10^{-3} \mathrm{~cm} \cdot \mathrm{sr}$ for higher energy electrons.

\section{Experimental results}

Some results of observations made by the time-of-flight instruments EM-1-1 and EM-1-2 (hereafter TOF analyzers) are presented here. Only some cases of the observed phenomenon are presented, when the upward high-energy electron beams were easily identifiable and of long duration. There are other cases, not included here, when the beam identification was shorter, or less reliable. The EA analyzers data as well as data from other onboard instruments are now being used for a more detailed analysis of those cases, and will be reported elsewhere.

During a satellite spin period the field-of-view of each TOF analyzer scans a complete circle in a plane perpendicular to the satellite rotation axis (which is directed towards the Sun). Depending on the angle

Table 1. Ranges of energy intervals for EM-1-1 and EM-1-2

\begin{tabular}{lcccc}
\hline Interval & \multicolumn{4}{l}{ Energy ranges, keV } \\
\cline { 2 - 5 } & $\mathrm{e}$ & $\begin{array}{l}\mathrm{M}=1 \\
\left(\mathrm{p}^{+}\right)\end{array}$ & $\begin{array}{l}\mathrm{M}=4 \\
(\alpha)\end{array}$ & $\begin{array}{l}\mathrm{M}=16 \\
\left(O^{+}\right)\end{array}$ \\
\hline 1 & $20-40$ & $30-50$ & $35-55$ & $55-90$ \\
2 & $40-70$ & $50-85$ & $55-90$ & $90-130$ \\
3 & $70-110$ & $85-115$ & $90-130$ & $130-185$ \\
4 & $110-150$ & $115-170$ & $130-185$ & $185-245$ \\
5 & $150-220$ & $170-225$ & $185-240$ & $245-315$ \\
6 & $220-290$ & $225-300$ & $240-315$ & $315-395$ \\
7 & $290-390$ & $300-395$ & $315-415$ & $395-510$ \\
\hline
\end{tabular}


between the satellite rotation axis and the magnetic field line, each analyzer scans a limited pitch-angle range $90^{\circ} \pm \varphi, \varphi \leq 90^{\circ}$, where $\varphi=90^{\circ}$ is a rather rare case. If a particle beam is more or less field-aligned, monodirectional and crosses the rotating field-of-view, it will be seen by the instrument once in a period. Then, as fieldsof-view of the two analyzers point in opposite directions, that beam will be seen by both instruments once in a period but with a half-period shift.

Below we have used for comparisons some preliminary data from the ION onboard particle spectrometer (courtesy of J.-A. Sauvaud and R. Kovrazhkin, see Sauvaud et al., this issue), and data from the onboard thermal mass-spectrometer HYPERBOLOID (courtesy of N. Dubouloz).

\subsection{Overall data description}

Case 1: pass 218 (see Fig. 1). The first of the events presented here was detected during the northern polar cap crossing on the 20 October, 1996. At 12:00 UT the satellite location was near the apogee (altitude about $19200 \mathrm{~km}$ ). The invariant latitude ILAT $=78.0^{\circ}$, magnetic local time MLT $=6 \mathrm{~h}$ at 12:00 UT, then the position changed to ILAT $=74.8^{\circ}, \mathrm{MLT}=7 \mathrm{~h}$ at $12: 30$ UT. The $K p$ value during the beam observation was 2 , Dst index was about $-30 \mathrm{nT}$. This pass took place during a quieting after a long disturbed period. The data collected by both TOF analyzers revealed the presence of a beam of $20-40 \mathrm{keV}$ electrons at large pitch angles (up to $175^{\circ}$, so the beam moved upward from the Earth). The beam was rather narrow, the angular width of its most intensive portion was about $\pm 20^{\circ}$. Maximum beam intensity reached more than $0.5 \cdot 10^{3}$ electrons $/ \mathrm{s} \cdot \mathrm{sr} \cdot \mathrm{keV} \cdot \mathrm{cm}^{2}$ at $175^{\circ}$ pitch angle, while at $90^{\circ}$ it was not greater than 4 electrons $/ \mathrm{s} \cdot \mathrm{sr} \cdot \mathrm{keV} \cdot \mathrm{cm}^{2}$

The closest ground stations from which magnetic field data were available at that time were Fort Simpson (Latitude $=61.8^{\circ}$, Longitude $=238.8^{\circ}$, MLT $\sim 3 \mathrm{~h}$ at 12:00 UT) and Leirvogur (Latitude $=64.2^{\circ}$, Longitude $=338.3^{\circ}$, MLT $\sim 10 \mathrm{~h}$ at 12:00 UT). The magnetogram from Fort Simpson indicated a series of a moderate substorm-like bursts from 03:00 to 10:30 UT, and the moment of our observations corresponds to a quieting period or a recovery phase of a small substorm. The magnetogram from Leirvogur also indicated a disturbed period from 00:00 to 08:00 UT, and then quieting. The solar wind and IMF data from the WIND spacecraft showed southward turning of the IMF $B z$ at the time corresponding to our observations (delayed by $22 \mathrm{~min}$ according to the solar wind velocity). Previous three hours of data indicated frequent southward turnings of the IMF $B z$ (WIND position at 11:40 UT was $X_{G S E}=112.4 \mathrm{R}_{\mathrm{E}}$, $Y_{G S E}=-69.8 \mathrm{R}_{\mathrm{E}}, Z_{G S E}=-1.6 \mathrm{R}_{\mathrm{E}}$, solar wind velocity $V x=-535 \mathrm{~km} / \mathrm{s}$ ).

The beam detection lasted for $15 \mathrm{~min}$ (i.e. for eight satellite rotation periods), with distinct field-aligned peaks and threshold fluxes around $90^{\circ}$ pitch angle. Then at 12:17 UT both TOF instruments detected a 3-min interval with a burst of rather isotropic flux with intensity of $10^{2} \div 10^{3}$ electrons $/ \mathrm{s} \cdot \mathrm{sr} \cdot \mathrm{keV} \cdot \mathrm{cm}^{2}$ at 20 $40 \mathrm{keV}$. The isotropic burst gradually decayed and the flux returned to the field-aligned beam which was more intense than before the burst, and had some fine structure around $90^{\circ}$ pitch angle. Its registration lasted until 12:30 UT.

Simultaneous measurements made by the ION instrument onboard the Auroral Probe (see Sauvaud et al.,

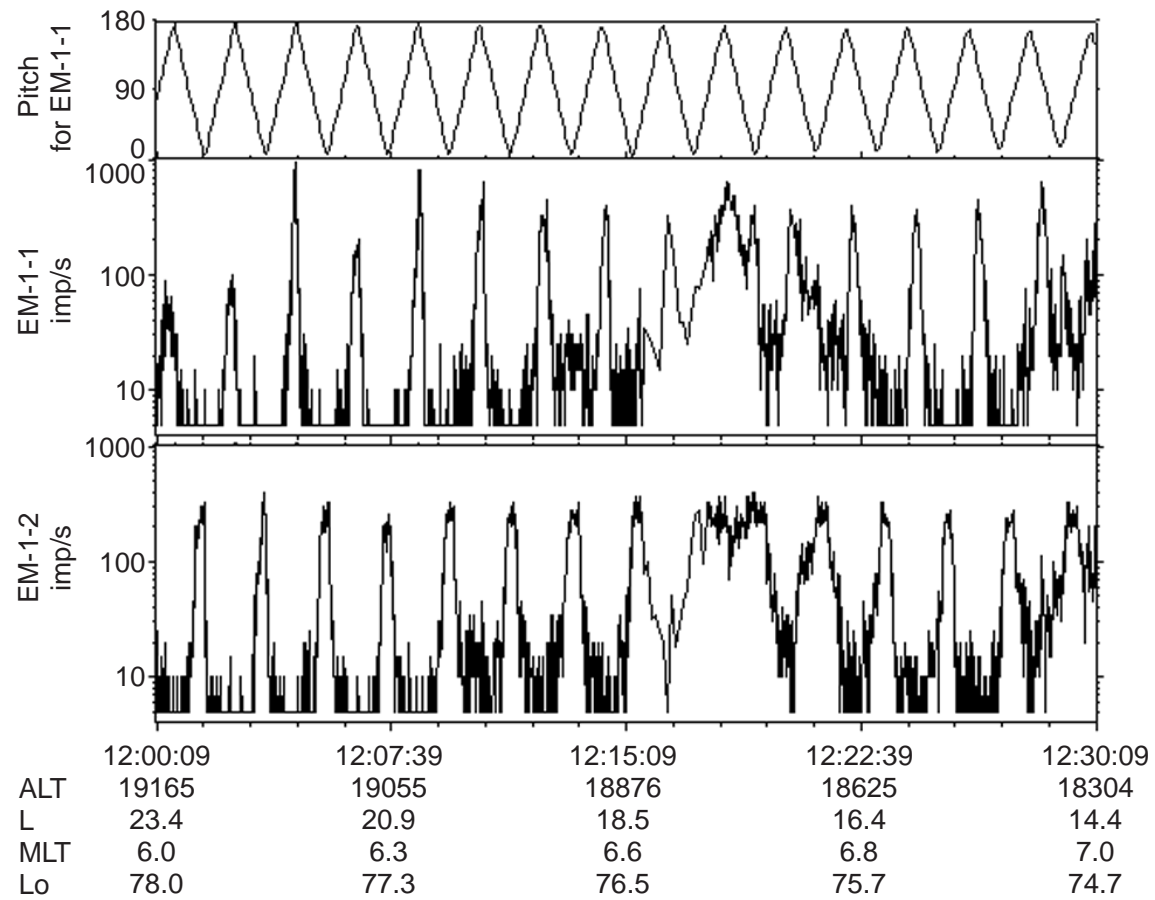

Fig 1. Case 1, October 20, 1996). Intensities of upward beam of electrons of $20-40 \mathrm{keV}$ by time-of flight spectrometers EM-1-1 and EM-1-2 in counts per second. In the top panel the pitch angle for EM-1-1 is shown (the pitch angle for EM-1-2 is shifted by half period in respect to that of EM-1-1, see Sect. 2 for details). The middle and lower panels show the 20-45 keV electrons data from EM-1-1 and EM-1-2, respectively. At the bottom there are also shown (from top to bottom): universal time; $A L T$ satellite altitude; $L$-shell; $M L T$, magnetic local time, and $L_{0}$, invariant latitude. 
this issue) indicated presence of a combination of an isotropic plasmasheet-type background intensity with intermittent FA counterstreaming electron beams in the energy range of $100 \div 1000 \mathrm{eV}$, at the time of the upward electron beam observations from the SKA-3. Also a beam of upward accelerated ions $\left(\mathrm{H}^{+}\right.$and $\left.\mathrm{O}^{+}\right)$ of $100 \div 1000 \mathrm{eV}$ energy was seen from 11:30 till 12:34 UT. At 12:17 UT, simultaneously with the isotropization burst observed by SKA-3, the ION spectrometer detected a 3-minute interval of isotropic flux of electrons in the energy range of $100 \div 1000 \mathrm{eV}$. The velocity dispersed burst of $\mathrm{H}^{+}$ions was detected at 12:17 UT in the energy range of $800 \mathrm{eV}-10 \mathrm{keV}$, along with the upward beam of $\mathrm{H}^{+}$in the range of $100 \div 1000 \mathrm{eV}$. The superthermal ion data available (thermal mass-spectrometer HYPERBOLOID) did not show any significant features which could be correlated with the simultaneously observed upward high-energy electron beam.

Case 2: pass 247 (see Fig. 2). At 11:00 UT on 27 October, 1996 the satellite position was ILAT $=79.1^{\circ}$ and $\mathrm{MLT}=2 \mathrm{~h}$, altitude about $17800 \mathrm{~km}$. The satellite was moving to the apogee (altitude about $19000 \mathrm{~km}$ ), at 11:30 UT it reached ILAT $=79.3^{\circ}$ and $\mathrm{MLT}=4 \mathrm{~h}$. During this time interval both TOF analyzers detected an upward beam of 20-40 keV electrons with intensity of about 10 electrons $/ \mathrm{s} \cdot \mathrm{sr} \cdot \mathrm{keV} \cdot \mathrm{cm}^{2}$ at pitch angles of $130^{\circ} \pm 20^{\circ}$ since 11:00 UT. The flux around $90^{\circ}$ was below the threshold. At 11:10 UT both instruments simultaneously detected a 3-min isotropic intensity burst with an intensity up to $0.5 \cdot 10^{3}$ electrons $/ \mathrm{s}$. $\mathrm{sr} \cdot \mathrm{keV} \cdot \mathrm{cm}^{2}$. Then instruments continued to detect the upward electron beam, now with an intensity reaching $0.6 \cdot 10^{2} \div 10^{3}$ electrons $/ \mathrm{s} \cdot \mathrm{sr} \cdot \mathrm{keV} \cdot \mathrm{cm}^{2}$, and a fine structure at pitch angles of $90^{\circ}$ (intensity there was about 10 electrons $\left./ \mathrm{s} \cdot \mathrm{sr} \cdot \mathrm{keV} \cdot \mathrm{cm}^{2}\right)$.
Simultaneous ION spectrometer measurements showed counterstreaming electron beams in the energy range of $40 \div 200 \mathrm{eV}$ after 11:10:30 UT and upward FA accelerated $\mathrm{O}^{+}$ions of $\sim 100 \mathrm{eV}$ after 11:11 UT. At 11:10:30 UT $\quad\left(\right.$ ILAT $\left.=79.5^{\circ}, \mathrm{MLT}=2.8 \mathrm{~h}\right)$ a 3 -min isotropic intensity burst of electrons of $100 \div 1000 \mathrm{eV}$ energy was seen, and at 11:11 UT a 3-min isotropic burst of intensity of $\mathrm{H}^{+}$ions of $400 \div 5000 \mathrm{eV}$ was detected. Before 11:10:30 UT the flux of ions in all energy ranges observed by ION was almost below the threshold, while the electron flux was detectable only in the range below $500 \mathrm{eV}$. From 11:05:30 UT till 11:09:30 UT, counterstreaming electron fluxes were seen in the range of $80 \div 500 \mathrm{eV}$. Unfortunately, for this case there were no superthermal ion observations.

Ground magnetometer data from Fort Simpson $(\mathrm{MLT} \sim 2.1 \mathrm{~h}$ at 11:10 UT) indicated a local substorm from 07:30 to 10:30 UT, so the upward electron beam was detected during the recovery phase of that substorm. WIND IMF and solar wind data indicated a decrease of proton number density (from $25 \mathrm{~cm}^{-3}$ to 12 $\mathrm{cm}^{-3}$ ) along with an increase of IMF $|B|$ magnitude and northward turning of the IMF $B z$ approximately at the moment corresponding to our observations (delayed according to solar wind velocity by $35 \mathrm{~min}$ ). From 08:00 to 12:00 UT there were two broad peaks of the solar wind proton number density up to $30 \mathrm{~cm}^{-3}$ separated by a period of lower density from 10:35 to 11:15 UT (average proton number density during the day was about $10 \mathrm{~cm}^{-3}$ ). The WIND position at 10:40 UT was $X_{G S E}=121.7 \mathrm{R}_{\mathrm{E}}, Y_{G S E}=-57.2 \mathrm{R}_{\mathrm{E}}, Z_{G S E}=1.9 \mathrm{R}_{\mathrm{E}}$, solar wind velocity $V x=-369 \mathrm{~km} / \mathrm{s}$. Value of $K p$ at the moment of observations was $1+$, Dst index was $-8 \mathrm{nT}$. Case 3: pass 250 (see Fig. 3). At 03:55 UT on 28 October, 1996 both TOF analyzers indicated a weak (less than 10 electrons $/ \mathrm{s} \cdot \mathrm{sr} \cdot \mathrm{keV} \cdot \mathrm{cm}^{2}$ ) beam of

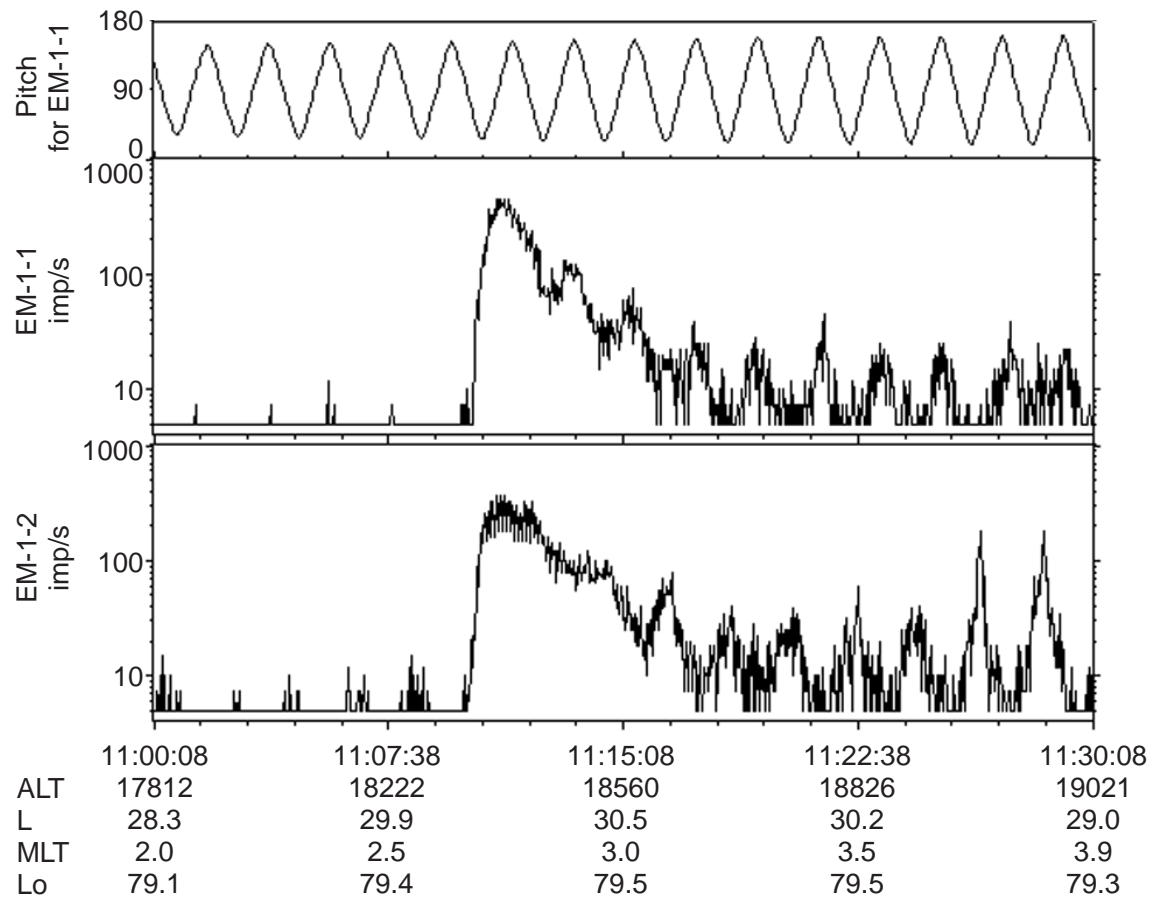

Fig 2. (Case 2, October 27, 1996). For details see Fig. 1 


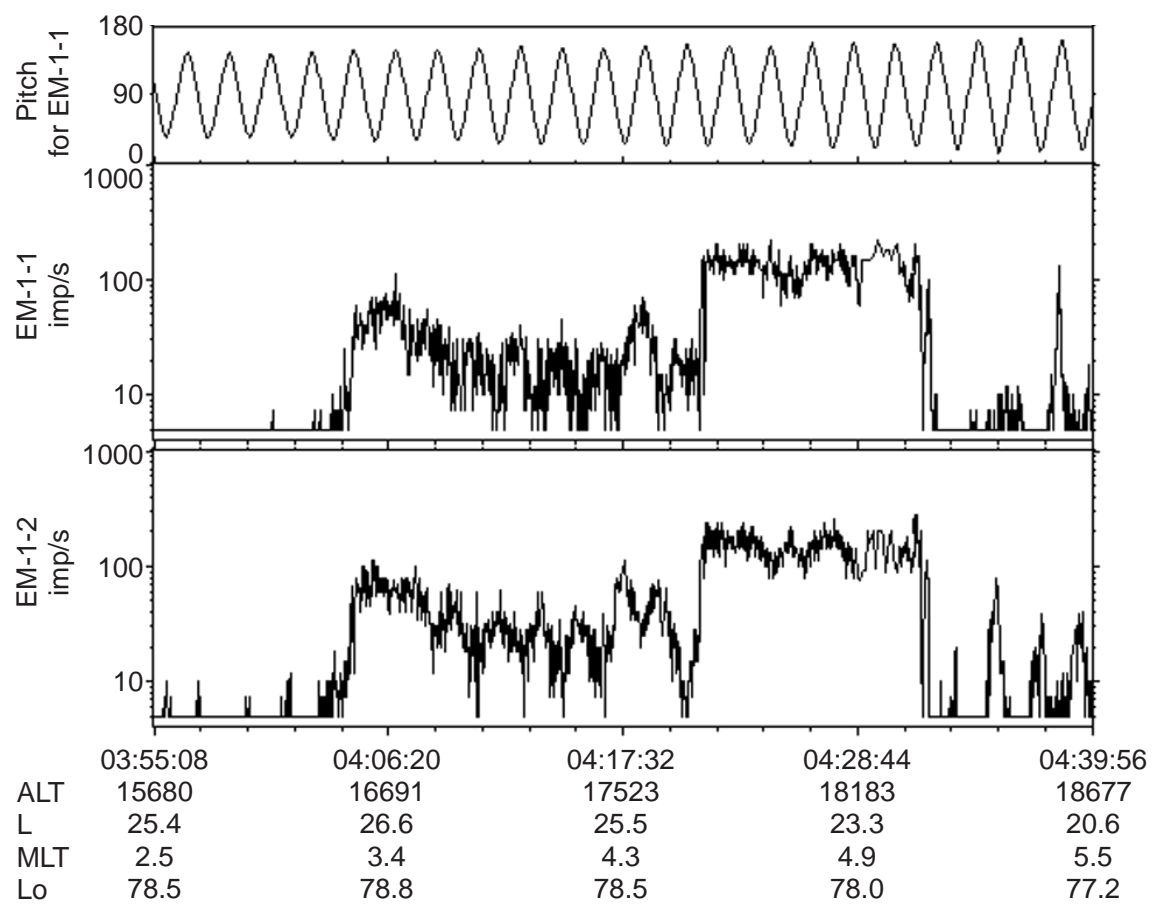

Fig 3. (Case 3, October 28, 1996). For details see Fig. 1
20-40 keV electrons a pitch angles about $130^{\circ} \pm 20^{\circ}$, with almost no counts above the threshold around $90^{\circ}$ of pitch angle. At 04:03 UT the TOF instruments detected a 3-min isotropic burst with an intensity of $0.5 \cdot 10^{2}$ electrons $/ \mathrm{s} \cdot \mathrm{sr} \cdot \mathrm{keV} \cdot \mathrm{cm}^{2}$. Then till 04:21 UT an upward electron beam was seen with peaks of the same intensity at pitch angles of $125^{\circ} \pm 25^{\circ}$. Around $90^{\circ}$ the observed flux was fine-structured, while the intensity did not exceed 4 electrons $/ \mathrm{s} \cdot \mathrm{sr} \cdot \mathrm{keV} \cdot \mathrm{cm}^{2}$.

At 04:21 UT both TOF analyzers detected an 11-min isotropic intensity burst with intensities up to $0 \cdot 5 \cdot 10^{3}$ electrons $/ \mathrm{s} \cdot \mathrm{sr} \cdot \mathrm{keV} \cdot \mathrm{cm}^{2}$ with some fine structure. After that until 04:39 UT an upward electron beam was seen with rather distinct peaks at pitch angles of $135^{\circ} \pm 15^{\circ}$ (intensity $0.5 \cdot 10^{3}$ electrons $/ \mathrm{s} \cdot \mathrm{sr} \cdot \mathrm{keV} \cdot \mathrm{cm}^{2}$ ). At $90^{\circ}$ the intensity was rather weak-number flux not greater than 10 electrons $/ \mathrm{s} \cdot \mathrm{sr} \cdot \mathrm{keV} \cdot \mathrm{cm}^{2}$. Other particle instruments of the Auroral Probe either were not operating at this time or the data is still unavailable.

The satellite position at 03:55 UT was $15650 \mathrm{~km}$ altitude, $\mathrm{MLT}=2.5 \mathrm{~h}$ and $\mathrm{ILAT}=78.5^{\circ}$; by $04: 39 \mathrm{UT}$ the satellite moved to an altitude of $18600 \mathrm{~km}$, $\mathrm{MLT}=5.5 \mathrm{~h}$ and ILAT $=77.3^{\circ} . K p$ value was $3-$, Dst was about $-3 \mathrm{nT}$. The magnetogram from the nearest ground station Leirvogur (MLT $\sim 3.5 \mathrm{~h}$ ) indicated a moderate disturbance or possible recovery phase of a small substorm. The WIND data indicated southward turning of the IMF $B z$ at the moment roughly corresponding to the observation time, shifted by the delay calculated from the solar wind velocity $(26 \mathrm{~min})$. The WIND position at 10:40 UT was $X_{G S E}=122.1 \mathrm{R}_{\mathrm{E}}$, $Y_{G S E}=-55.5 \mathrm{R}_{\mathrm{E}}, Z_{G S E}=2.3 \mathrm{R}_{\mathrm{E}}$, solar wind velocity $V x=-500 \mathrm{~km} / \mathrm{s}$.

Case 4: pass 251 (see Fig. 4). The last case we consider here took place at 10:12 UT on 28 October, 1996. After 10:12 UT for $40 \mathrm{~min}$ the TOF analyzers detected an upward beam of $20-40 \mathrm{keV}$ electrons with intensities up to $0.5 \cdot 10^{2}$ electrons $/ \mathrm{s} \cdot \mathrm{sr} \cdot \mathrm{keV} \cdot \mathrm{cm}^{2}$, pitch angles about $135^{\circ} \pm 15^{\circ}$. Then at 10:52 UT (ILAT $=79.1^{\circ}, \mathrm{MLT}=4.8 \mathrm{~h}$ ) some flux intensification began, up to $0.5 \cdot 10^{3}$ electrons $/ \mathrm{s} \cdot \mathrm{sr} \cdot \mathrm{keV} \cdot \mathrm{cm}^{2}$, along with isotropization and intensification of flux around pitch angles of $90^{\circ}$. From 11:16:50 to 11:23:45 UT $\left(76.9^{\circ}<\right.$ ILAT $<76.2^{\circ}, \quad 5.8<$ MLT $\left.<6.0 \mathrm{~h}\right)$, distinct peaks at maximum pitch angles were seen and almost no detectable flux at $90^{\circ}$ of pitch angle, after that a 3min isotropic burst was seen.

Other particle instruments observations are available only after 11:00 UT. The ION particle spectrometer detected a rather isotropic flux of $100 \div 1000 \mathrm{eV}$ electrons until 11:13 UT. Then ION detected a series of isotropic $100 \div 1000 \mathrm{eV}$ electron bursts: from 11:16 till 11:17, from 11:19 till 11:21, from 11:24 till 11:27 UT. After that the electron flux became isotropized. Several velocity dispersed $\mathrm{H}^{+}$structures below $8 \mathrm{keV}$ were also detected by ION from 11:03 till 11:16 UT, and then after 11:24 UT. An isotropic $\mathrm{H}^{+}$burst in the energy range $100 \div 3000 \mathrm{eV}$ was detected by ION from 11:16 till 11:18 UT. In addition, an upward $100 \mathrm{eV} \mathrm{O}^{+}$beam was detected from 11:00 till 11:53 UT. The measurements of the thermal plasma analyzer HYPERBOLOID showed an upward beam of $10 \mathrm{eV} \mathrm{H}^{+}$from 11:00 till 11:55 UT, slightly isotropized from 11:37 till 11:45 UT.

From the WIND data for the case an unstable IMF $B z$ behavior is seen: there was a 10 -min southward IMF $B z$ turning near the moment corresponding to the observation time shifted by the appropriate delay (22 min). WIND position at 10:40 UT was $X_{G S E}=122.1 \mathrm{R}_{\mathrm{E}}, Y_{G S E}=-54.7 \mathrm{R}_{\mathrm{E}}, Z_{G S E}=2.5 \mathrm{R}_{\mathrm{E}}$, solar wind velocity $V x=-585 \mathrm{~km} / \mathrm{s}$. The Auroral Probe position was changing from $18000 \mathrm{~km}$ altitude, MLT $=2 \mathrm{~h}$ and ILAT $=80^{\circ}$ to $18200 \mathrm{~km}$, MLT $=6.2 \mathrm{~h}$ and 


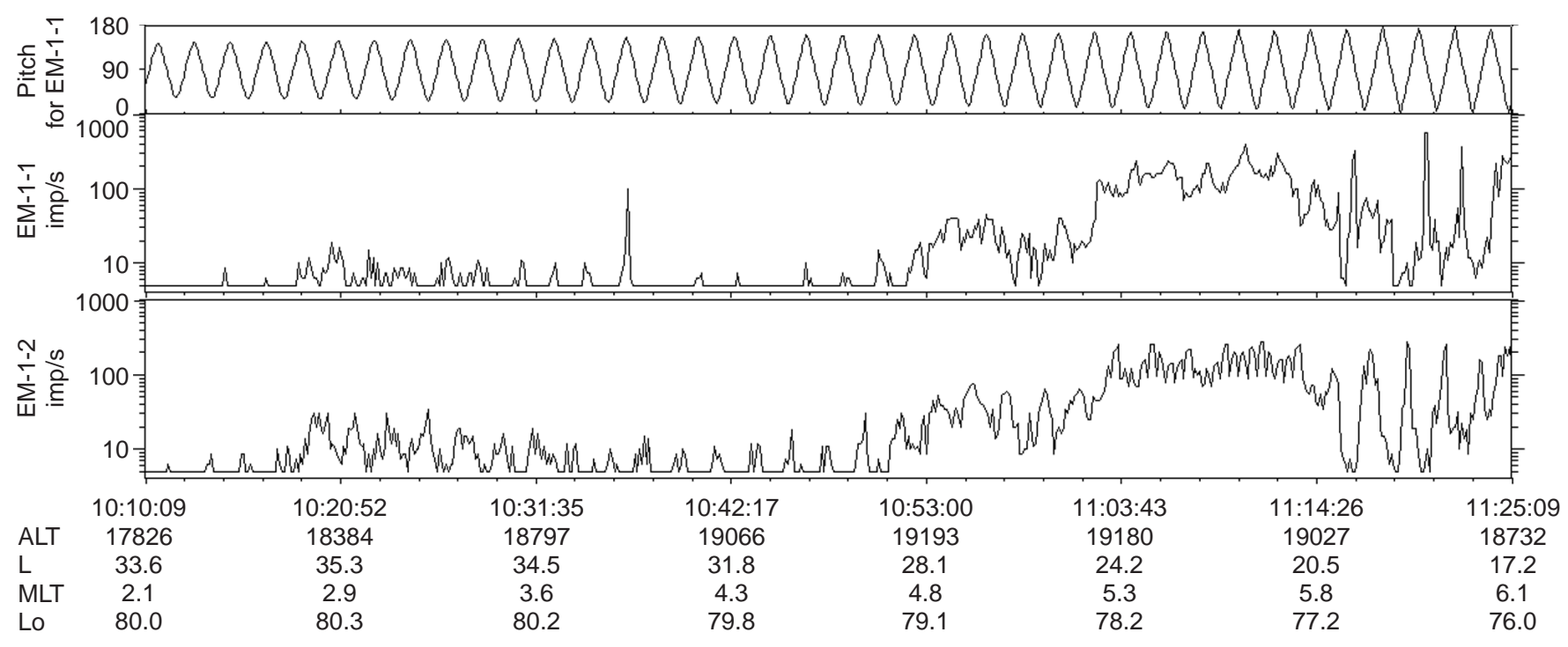

Fig 4. (Case 4, October 28, 1996). For details see Fig. 1

ILAT $=75^{\circ}$. During the beam registration the satellite's orbit crossed its apogee at 11:03 UT, MLT $=5.4 \mathrm{~h}$, ILAT $=78.5^{\circ}$. At the moment of observations $K p$ was 3-, Dst about -22 nT. Ground magnetometer data from College $\quad$ (Latitude $=64.8^{\circ}, \quad$ Longitude $=212.2^{\circ}$, MLT $\sim 0 \mathrm{~h}$ ) and Fort Simpson (MLT $\sim 1.3 \mathrm{~h}$ ) indicated that the beam was detected during a quieting period between two substorm-like bursts, or just before the breakup phase of a local activation.

\subsection{Observations summary}

As can be seen from the data description, the four cases detected were located from 2 to $7 \mathrm{~h}$ MLT and from $75^{\circ}$ to $80^{\circ}$ ILAT near the apogee of the Auroral Probe orbit (i.e. at the polar edge of the auroral oval and/or inside the northern polar cap), during quieting, or moderate disturbance levels. ION particle spectrometer measurements onboard the Auroral Probe (Sauvaud et al., 1997, this issue) for Case 2 also show that the satellite probably crossed the poleward auroral oval boundary during, or before, the beam registration. Observations revealed the existence of narrow field-aligned upward beams of energetic electrons in that region interrupted by isotropic intensity burst of about 3 min duration, after which the upward beam intensity increased. At the same time, in the $\mathrm{keV}$ energy range, counterstreaming FA electron beams of variable intensity (generally much higher than that of the upward beam of $20-40 \mathrm{keV}$ ) were seen simultaneously with nearly isotropic intensity of plasmasheet-like electrons. Sometimes upward beams of low-energy ions (probably, conics narrowed in pitch angle due to magnetic moment conservation) were seen more or less simultaneously with the upward highenergy electron beam. From a limited set of the data available it is not clear whether the presence of such energetic upward electron beams is caused by some distinct signature in the IMF and solar wind. Cases 1, 3 and 4 probably correspond to the southward turnings of the IMF $B z$, but Case 2 seems to be detected during northward turning of the IMF $B z$. Global magnetospheric conditions were not very disturbed, as is shown by $K p$ and $D s t$ values, and loosely could be described as quieting periods. Ground magnetograms from the nearby stations correspond to intervals after or between disturbances of moderate activity levels, or probably to a recovery or late recovery phase of a small substormlike bursts. There is no clear evidence that the events observed are a product of a preceding substorm activity. However this is not inconsistent with the data available.

Simultaneously with the energetic upward beam observations by SKA-3, measurements performed by the ION particle instrument show variable counterstreaming electron beams of $100 \div 1000 \mathrm{eV}$ embedded in the plasmasheet-like background. An isotropic but finestructured intensity bursts of $100 \div 1000 \mathrm{eV}$ electrons and $\mathrm{H}^{+}$ions of up to some $\mathrm{keV}$ energy were observed after several minutes of the upward beam appearance at least for Cases 1 and 2. In addition an upward accelerated ion beam of $\mathrm{keV}$ energy was observed in Case 1, as well as a set of velocity-dispersed $\mathrm{H}^{+}$structures below 8 $\mathrm{keV}$ and upward $100 \mathrm{eV} \mathrm{O}^{+}$beam for Case 4. An upward beam of $10 \mathrm{eV} \mathrm{H}^{+}$was also detected by the thermal mass-spectrometer HYPERBOLOID in Case 4.

Further studies of features accompanying the upward high-energy electron beams require detailed analysis of simultaneous observations performed by other particle detectors onboard the Auroral Probe such as ION, PROMICS-3 and DOK-2, as well as wave and electric field instruments and onboard magnetometers. The work of data collection and analysis during the upward high-energy electron beam registrations and some modeling efforts are under way and will be reported elsewhere.

In the next section we propose a qualitative scenario of the mechanism which could be responsible for the 
formation of energetic upward electron beams in that ILAT-MLT region above the polar ionosphere.

\section{An interpretation scenario}

\subsection{Features of the upward high-energy electron beams}

In an attempt to construct a plausible theoretical scheme for explanation of the observed FA upward beams of energetic electrons, the following morphological properties of the phenomenon will be taken into account:

1. The high-energy beams $(20-40 \mathrm{keV})$ are observed for a relatively long time $\left(\sim 3 \cdot 10^{2}-10^{3} \mathrm{~s}\right)$, when the satellite stays almost at the same place, or moves slowly in the ILAT-MLT frame. Thus they may be considered as quasi-stationary.

2. The high-energy FA upward electron beam is nearly monodirectional, while the simultaneously present electron FA beams of $\mathrm{keV}$ energy are observed as bidirectional and highly variable. In some cases highenergy electron fluxes at $90^{\circ}$ also appear, but are much weaker than the FA upward beam.

3. The ILAT-MLT location of the beams at, or close to, the polar border of the oval in the postmidnightmorning MLT sectors is consistent with a region of large-scale downward FA current (region I). But the preliminary onboard magnetometer data (courtesy of V. Styazhkin, IMAP-3 experiment) are inconclusive to prove the existence of the downward current. Recent results by FAST group (see, e.g., Carlson et al., 1998, and references therein) show high wave activity and intermittent small-scale potential drops in the downward current region. The inference about the downward FA current at the upward beam locations is also consistent with the "Matreshka" scheme of nested FA current loops at the polar edge of the postmidnight oval (Timofeev and Galperin, 1991).

4. Simultaneous but preliminary data on ELF plasma waves (courtesy of M. Mogilevsky, IESP instrument) indicate that while some wave activity is usually present during an event, no straightforward correlations have been found so far.

\subsection{A qualitative scenario}

In view of these characteristics, a scenario for the highenergy acceleration phenomenon is proposed. In this scenario, during registration of the upward energetic electron beam the satellite is supposed to be within a bidirectional electron acceleration region, or at least, the FA acceleration of electrons occurs both above and below the satellite. This is evidenced by the counterstreaming low-energy electron beams embedded in the plasmasheet-like particle environment. Thus the FA low-energy electrons $(\sim 0.1 \div 1 \mathrm{keV})$, reaching the satellite from both directions, have been accelerated by some wave-particle interactions while crossing a part of the acceleration region lying respectively below or above the satellite ("single transit").

The upward beam of high-energy electrons is presumed here to originate from the acceleration of a part of the FA accelerated plasmasheet electrons. Before the formation of the upward beam, those electrons have passed downward through the whole acceleration region both above and then below the satellite ("double transit"). As a result of the FA acceleration they formed electron beams, mostly reaching auroral altitudes, with excitation of respective auroral phenomena, probably in form of diffuse (or weakly structured) features, sometimes with additional activation. The portion of the accelerated electron distribution lying outside the loss cone, after being mirrored below the acceleration region (but above the ionosphere), and now moving upward, is able to reach the satellite again after passing the lower part of the acceleration region ("triple transit"). Thus particles that formed an upward beam of high-energy electrons have experienced much more effective acceleration in a "triple transit" than those registered at the satellite as counterstreaming electron beams which experienced only a "single transit", or auroral electrons at the footprint of the satellite that have experienced a "double transit" through the region of stochastic waveparticle acceleration within the downward FA current region. This scenario is shown schematically in Fig. 5.

\subsection{On possible acceleration mechanisms}

Consider now a possible mechanism for the particle acceleration process. For the scenario discussed it must be bidirectional, i.e., in these wave-particle interactions,

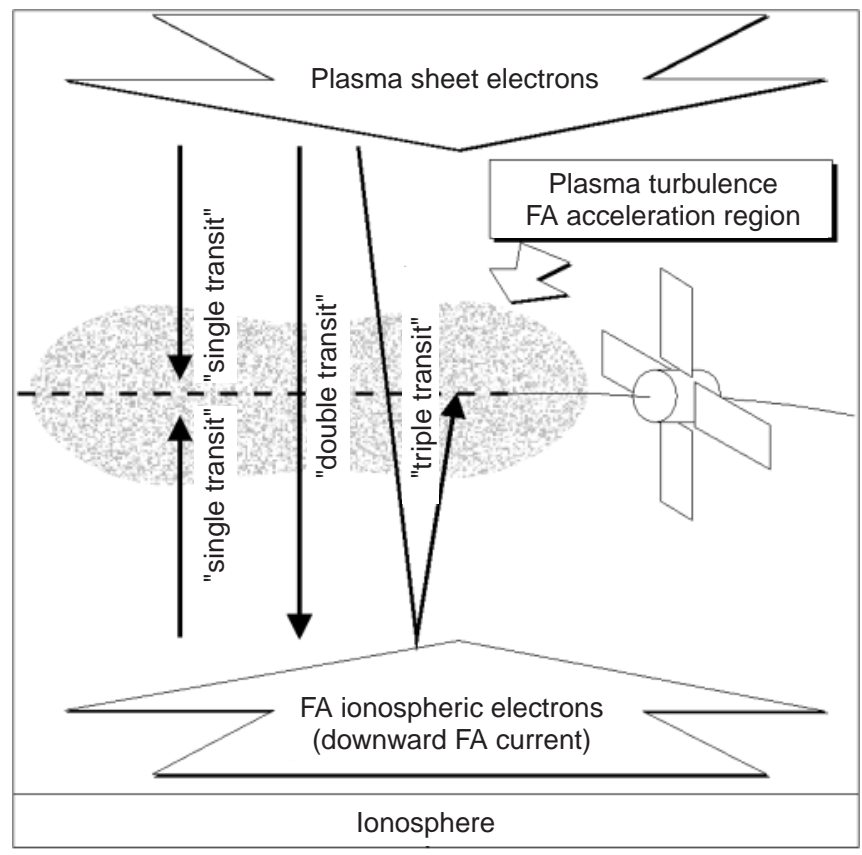

Fig 5. Schematics of the acceleration scenario 
the acceleration is experienced by electrons moving either upward or downward through the layer of wave-particle interactions. For that, a stochastic type of FA acceleration is suitable if accomplished by waves with FA electric field components in either direction. At the same time, as no comparable acceleration effects are observed for ions, and considering the available wave and other particle data onboard, some restrictions probably may be devised for the choice of the wave-particle interactions involved. This work is in progress now.

According to our observations, the acceleration by wave-particle interactions is present in a wide range of altitudes along the particle trajectory. It occurs both above and below the satellite and is not confined in the altitude region of $0.5-2 \mathrm{R}_{\mathrm{E}}$, where most of the auroral FA acceleration takes place. It is not clear at what altitudes above the satellite the FA downward acceleration of the plasmasheet electrons occurs. It must be confined at most to $\sim 6 \mathrm{R}_{\mathrm{E}}$, because otherwise the magnetic moment conservation tendency will isotropize the electron distribution more than is observed. At the same time, the observed weakness of the high-energy electron intensities around $90^{\circ}$ pitch angle in comparison to the FA component indicates that the perpendicular acceleration process by plasma waves is not local. Thus, we cannot expect a good correlation of the upward beam intensity with some particular type of waves registered by the Auroral Probe. A search for such waves, both from measurements on the Auroral Probe and from theoretical analysis, is in progress.

Turbulent acceleration processes in the aurora have been discussed in many papers (see, e.g., Bryant et al., 1991; Tetreault et al., 1995), however, no definite comparisons with the data are known so far. Intense IC and LH wave packets and their interaction with particles have been studied using a kinetic approach by many authors (see, e.g., Andre and Chang, 1992; Kintner et al., 1992; Retterer et al., 1994; and recently Dubouloz et al., 1995 for LHR wave packets). Supposing the initial plasmasheet electron temperature of 0.5 $\mathrm{keV}$, model results of Dubouloz et al., 1995, show the possibility for such electrons to be FA accelerated up to several $\mathrm{keV}$ due to $\mathrm{LH}$ wave packets in a single particle transit through the upper part of the acceleration region (above the satellite), or through its lower part (below the satellite), if LH waves of sufficient intensity are present there. These accelerated electrons will then constitute the observed FA counterstreaming beams of about $1 \mathrm{keV}$. However, the resonance condition for high-energy electrons of the upward beam requires very large wave phase velocities hardly achievable by the LH wave packets. But much higher energies, up to 20 or more $\mathrm{keV}$, are observed to be attainable by a small part of the initial plasmasheet electron population which is presumed here to mirror above the ionosphere after traversing the whole accelerating layer, forming an upward accelerated energetic electron beam. Thus, the theory outlined already is unable to explain observations fully by itself and additional mechanisms are needed.

The described scenario seems to be qualitatively consistent with observations of the energetic upward electron beams as described, while the nature of the wave-particle interactions involved remains obscure. According to the scenario, the observed energetic upward beam is the extended tail of the FA accelerated electrons of lower energies, presumably with a power law spectral form. The accompanying feature expected from the scenario is a wave turbulence in extended regions somewhere above, and especially below the satellite, but not necessarily at its altitude. In addition, for the LHR wave packets e.g., a perpendicular ion heating and thus ion conic formation is another expected consequence. We are now studying the available data with respect to these inferences.

\section{Discussion}

\subsection{Location in magnetosphere and time development}

We have concentrated here on a few cases of long duration events when, during many satellite rotations (2 min each), both oppositely directed TOF analyzers indicated the presence of an upward high-energy electron beam. At this time no reliable statistics of such cases could be presented.

The four cases described occur in the postmidnightmorning sector of MLT near, or at, the polar edge of the auroral oval, or within the polar diffuse auroral zone (see, Feldstein and Galperin, 1985, 1996; Galperin and Feldstein, 1991). These magnetic flux tubes in stationary conditions are expected to be traced to, or close to, the open-closed magnetic field boundary, or the distant reconnection line. A suggestion about an extended region in the distant tail with turbulent magnetic field as an alternative to the single distant reconnection line was recently put forward by Galperin, (1995) and Galperin and Feldstein 1996. However, the conditions in which the four events studied here took place cannot be considered as stationary states of the magnetosphere. They all belong to some quieting period after, or between, substorm-like disturbances or activations, when the distant tail still could not be equilibrated, and filaments of plasma with different properties could extend from the plasmasheet to tail lobes, or plasmoid(s) in the tail could be still present, causing variable FA currents in the regions of beam observations. As there is no returning high-energy electron beam in these cases, a quasi-regular closed magnetic field along the beam plasma flux tube is excluded (in contrast to the late morning case described by Hultqvist et al., 1988). Thus, either an open magnetic field, or a turbulent weak magnetic field in the distant tail where strong scattering of the high-energy electrons occurs, seem to be the alternatives for these plasma flux tubes.

Now let us consider the time development of the observed energetic electron beams and ambient plasma populations. In the four cases described qualitatively a very similar behavior was found: a weak upward beam of 20-40 keV electrons after several minutes was followed by an isotropic intensity burst that lasted for about 3 min. It occurred simultaneously with the 
isotropic burst of electrons and ions in the keV energy range. After the burst the quasi-steady high-energy electron beam reappeared and was more intense than before the burst. We note that the 3 min interval of the burst is a characteristic time for the transit of protons of $0.3-3 \mathrm{keV}$ from the distant tail region at $50-160 \mathrm{R}_{\mathrm{E}}$. Thus, the origin of the burst in some active processes in the far tail seems plausible, and deserves further analysis.

\subsection{A possible relation to energetic particle bursts in the distant tail}

The Auroral Probe orbit is chosen so that the satellite at the apogee moves only very slowly in the ILAT-MLT frame for a relatively long time $(\sim 20 \mathrm{~min})$, thus the time dependence of the events can be distinguished from the data more easily. This was the case during the registration of the upward electron beams. As could be seen from our case studies, there is each time a period of a relatively weak flux (as long as several satellite spin periods of $120 \mathrm{~s}$ ) before an intensity burst and isotropization interval followed by subsequent upward beam intensification.

Sarris et al., 1996, have reported Geotail observations of the high-energy electron and ion bursts in the deep magnetotail at a distance of $X_{G S M}=-128 \mathrm{R}_{\mathrm{E}}$. The source location of these high-energy particles is reported to be at $\sim-103 \mathrm{R}_{\mathrm{E}}$. Then it could be of the same type as the isotropic intensity bursts observed by the SKA-3 after some time of quasi-steady upward beam existence. However, no sign of the preceding weak high-energy beam intensity was noted in Sarris et al.'s 1996, study. We note that if the source region was in the closed field line region, or a reconnection region, such a location could be mapped to the ILAT-MLT region of our observations. Thus energetic particle bursts in the tail during the SKA-3 observations cannot be excluded, and at least, in some cases, such checks will be possible from simultaneous Tail Probe data.

\subsection{A speculation concerning possible local destabilization} of the cross-tail current by high-energy electrons of the upward beam

It is known that bursts of energetic electrons occur in the distant tail (see Lui and Krimigis, 1984; Sarris et al., 1996). It has been shown by Sitnov et al., 1996, that the loss cone electron population could affect significantly the linear stability conditions of the tearing mode in the quasi-neutral magnetotail current sheet. So it might be speculated from this limited data set that the initial upward energetic electron beam (even while rather weak) could affect the distant tail stability conditions, as the field lines on which the measurements were performed map to more than $-70 \mathrm{R}_{\mathrm{E}}$ geocentric according to the Tsyganenko-87 model. Supposedly, the induced local destabilization of the tearing mode in the distant tail plasma and currents could cause the observed isotropic burst of energetic particles which is detected by the SKA-3 instruments. Presumably the plasma turbulence level at altitudes below and above the satellite, which is responsible for the acceleration of high-energy electrons, increases after the particle burst, and qualitatively it can cause the upward beam intensification after the burst.

One possibility to significantly alter locally the crosstail current in the distant tail is to inject particles there with a Larmor radius comparable to the local magnetic field line curvature in the neutral sheet. According to the data from the ISEE-3 and GEOTAIL, the average magnetic field in the neutral sheet at $\sim 100 \mathrm{R}_{\mathrm{E}}$ is about 0.3 nT (Fairfield, 1992, 1993; Nishida et al., 1995; see also Lui and Krimigis, 1984) and the curvature radius can be less than $2 \mathrm{R}_{\mathrm{E}}$. For a $25 \mathrm{keV}$ electron the respective Larmor radius amounts then to about $1.7 \mathrm{R}_{\mathrm{E}}$, so that its strong scattering due to non-adiabatic motion is quite feasible. It may be supposed that, due to appearance of new charge carriers with a different type of particle motion in the cross-tail current sheet, the charge neutrality and cross-tail current characteristics could be locally disturbed. It is speculated here that this disturbance could lead to the plasma particle burst observed in the middle of the upward beam observations.

Clearly this point is just a speculation based on the medium-altitude measurements and requires further analysis and comparisons with the data from other satellites and ground-based observations.

\section{Summary and conclusions}

1. A quasi-stable upward field-aligned electron beam with super-auroral energies $(20-40 \mathrm{keV})$ was discovered at altitudes $\sim 3 \mathrm{R}_{\mathrm{E}}$, high above the post-midnight polar border of auroral oval (or at, or near, the open-closed magnetic field boundary) during quieting conditions.

2. From four case studies presented, the time development of the beam starts from a low monodirectional upward flux, but after 5-15 $\mathrm{min}$ an isotropic intensity burst appears, decays in $\sim 3 \mathrm{~min}$ and the upward monodirectional beam reappears with higher intensity than before the burst.

3. The appearance of bidirectional electron beams at altitudes $\sim 3 \mathrm{R}_{\mathrm{E}}$ during the upward high-energy electron beams demonstrates that electron FA acceleration processes, presumably by the wave-particle interactions, can encompass a very extended altitude range, up to the Auroral Probe apogee and higher, at least for the cases considered.

4. A qualitative scenario is proposed for FA acceleration of the tail of the plasmasheet electron distribution function both above and below the satellite altitude, by electron velocity diffusion due to unspecified waveparticle interactions. The scenario includes additional acceleration of the mirrored, upward moving, electrons, which gives them higher energy than those reaching auroral altitudes. 
5. A local destabilizating action is suggested from the high-energy FA electron beam for the cross-tail current in the distant tail plasma sheet as a possible cause of the isotropic intensity burst observed after several minutes of the upward beam persistence.

Acknowledgements. The Interball Project was accomplished with in the frame of the contract with the Russian Space Agency, N0257535/94. The work of V. Stepanov, Y. Galperin, F. Shuiskaya and A. Kuzmin was supported by grants from Russian Foundation for Basic Research (RFBR) N 94-02-04299 and 97-02-16333; and by grant INTAS N 94-1695. We thank Russian Space Agency, Lavochkin Space Association and Babakin Space Center for their continuous efforts which led to the success of the Interball project. We wish to thank N. Dubouloz, J.-J. Berthelier, A. Volosevich and L. Zelenyi for many valuable discussions, N. Dubouloz (HYPERBOLOID thermal mass-spectrometer PI), J.-A. Sauvaud and R. Kovrazhkin (ION particle instrument PIs), M. Mogilevsky and S. Perraut (IESP instrument Pis), V. Styazhkin (IMAP-3 onboard magnetometer PI) for their permission to use, respectively, the preliminary HYPERBOLOID, ION, IESP and IMAP-3 data in our analysis. Also we would like to thank R. Lepping and K. Ogilvie from NASA/GSFC, WIND magnetic field and solar wind experiments PIs, for the key magnetic field and solar wind parameters available at http://rumba.gsfc.nasa.gov/cdaweb/; and NGDC staff made available the ground magnetometers data from College, Fort Simpson and Leirvogur stations at the WWW site http://www.ngdc.noaa.gov:8080/production/html/GEOMAG/geo_search_frames.html.

Topical Editor K.-H. Glassmeier thanks H. E. J. Koskinen and $\mathrm{J}$. Retterer for their help in evaluating this paper.

\section{References}

Andre, M., and T. S. Chang, Ion heating perpendicular to the magnetic field, in Physics of Space Plasmas, Number 12, Eds. Chang, T. and J. R. Jasperse, Scientific Publishers, Inc., Cambridge, Mass, USA, 35-71, 1992.

Bryant, D. A., A. C. Cook, Z.-S. Wang, U. de Angelis, and C. H. Perry, Turbulent acceleration of auroral electrons, J. Geophys. Res., 96, 13 829-13 839, 1991.

Burch, J. L., P. H. Reiff, and M. Sugiura, Upward electron beams measured by DE-1: a primary source of dayside region-1 Birkeland currents, Geophys. Res. Lett., 10, 753-756, 1983.

Carlson, C. W., J. P. McFadden, R. E. Ergun, M. Temerin, W. Peria, F. S. Mozer, D. M. Klumpar, E. G. Shelley, W. K. Peterson, E. Moebius, R. Elphic, R. Strangeway, C. Cattell, and R. Pfaff, FAST observations in the downward auroral current region: energetic upgoing electron beams, parallel potential drops, and ion heating, Geophys. Res. Lett., 25, 2017-2020, 1998.

Dubouloz, N., R. A. Treumann, R. Pottelette, and K. A. Lynch, Electron acceleration from localized lowerhybrid waves, Geophys. Res. Lett., 22, 2969-2972, 1995.

Fairfield, D. H., On the structure of the distant magnetotail: ISEE 3, J. Geophys. Res., 97, 1403-1410, 1992.

Fairfield, D. H., Solar wind control of the distant magnetotail: ISEE 3, J. Geophys. Res., 98, 21 265-21 276, 1993.

Feldstein, Y. I., and Y. I. Galperin, The auroral luminosity structure in the high-latitude upper atmosphere: its dynamics and relationship to the large-scale structure of the Earth's magnetosphere, Rev. Geophys. Space Phys., 23, 217-275, 1985.

Feldstein, Y. I., and Y. I. Galperin, Structure of the auroral precipitations in the nightside sector of the magnetosphere, Cosmic Res. 34, 209-227, 1996.
Galperin, Y. I., Magnetospheric tail structure: concepts, problems, and storm-time development of the auroral oval, J. Atmos. Terr. Phys. 57, 1397-1414, 1995.

Galperin, Y. I., Polar magnetosphere during a solar minimum - a look from INTERBALL-2 (Abstr). Annales Geophysicae, 1.15 (3) C 678, 1997.

Galperin Y. I., and Y. I. Feldstein, Auroral luminosity and its relationship to Magnetospheric plasma domains. In Auroral Physics, Eds. Meng, C.-I., M. J. Rycroft, and L. A. Frank, Cambridge UP, Cambridge, 207-222, 1991.

Galperin, Y. I., and Y. I. Feldstein, Mapping of the precipitation regions to the plasma sheet, J. Geomag. Geoelectr., 48, 857-875, 1996.

Hultqvist, B., On the acceleration of electrons and positive ions in the same direction along magnetic field lines by parallel electric fields, J. Geophys. Res., 93, 9777-9784, 1988.

Hultqvist, B., R. Lundin, K. Stasiewicz, L. P. Block, P.-A. Lindkvist, G. Gustafsson, H. Koskinen, A. Bahnsen, T. A. Potemra, and L. J. Zanetti, Simultaneous observation of upward moving fieldaligned energetic electrons and ions on auroral zone field lines, J. Geophys. Res., 93, 9765-9776, 1988.

Kintner, P. M., J. L. Vago, S. W. Chesney, R. L. Amoldy, K. A. Lynch, C. J. Pollock, and T. E. Moore, Localized lower hybrid acceleration of ionospheric plasma, Phys. Rev. Lett., 68, 24482451, 1992.

Lui, A. T. Y., and S. M. Krimigis, Association between energetic particle bursts and Birkeland currents in the geomagnetic tail, $J$. Geophys. Res., 89, 10741-10748, 1984.

Nishida, A., T. Mukai, Y. Saito, S. Kokobun, and K. Maezawa, GEOTAIL observation of magnetospheric convection in the distant tail at $200 \mathrm{R}_{\mathrm{E}}$ in quiet times, J. Geophys. Res., 100, 23663-23675, 1995.

Retterer, J. M., T. S. Chang, and J. R. Jasperse, Transversely accelerated ions in the topside ionosphere, J. Geophys. Res., 99, 13189-13201, 1994.

Sarris, E. T., V. Angelopoulos, R. W. McEntire, D. J. Williams, S. M. Krimigis, A. T. Y. Lui, E. C. Roelof, and S. Kokobun, Detailed observations of a burst of energetic particles in the deep magnetotail by GEOTAIL, J. Geomag. Geoelectr. 48, 649656, 1996.

Shuiskaya, F. K., A. K. Kuzmin, Y. I. Galperin, V. A. Stepanov, R. A. Kovrazhkin, L. S. Gom, B. A. Ilyin, M. V. Iovlev, A. A. Klimashov, I. I. Cherkashin, B. I. Khazanov, and A. Y. Safronov, Measurements of charged particles by means of the experiment SKA-3, in: Interball Mission and Payload, Eds. Galperin, Y., T. Muliarchik, and J.-P. Thouvenin, RKA, IKI, CNES, Paris, 262-267, 1995.

Shuiskaya, F. K., Y. I. Galperin, R. A. Kovrazhkin, A. K. Kuzmin, V. A. Stepanov, L. S. Gorn, B. I. Khazanov, Measurements of energetic charged particles at high latitudes: the SKA-3 experiment on the Auroral probe (INTERBALL-2) satellite, in: Cosmic Res. 36, 81-91, 1998.

Sitnov, M. I. H. V. Malova, and A. S. Sharma, Role of the temperature ratio in the linear stability of the quasineutral sheet tearing mode, Geophys. Res. Lett., 25, 269-272, 1998.

Stepanov, V. A., F. K. Shuiskaya, A. K. Kuzmin, and A. A. Skalsky, Energetic particle beams at about $3 \mathrm{R}_{\mathrm{E}}$ above the high-latitude polar cap (Abstr.), Annales Geophysicae, 15(3), C 687, 1997.

Tetreault, D. J., Theory of electric fields in the auroral acceleration region, J. Geophys. Res., 96, 3549-3563, 1991.

Timofeev, E. E., and Y. I. Galperin, Convection and currents in stable auroral arcs and inverted-V's. J. Geomag. Geoelectr., 43, Suppl., 259-274, 1991. 